\title{
Streptococcus suis: an important zoonotic pathogen for human - prevention aspects
}

\author{
VG Papatsiros*¹, D Vourvidis², AA Tzitzis ${ }^{3}$, PS Meichanetsidis ${ }^{3}$, D Stougiou ${ }^{4}$, \\ D Mintza ${ }^{4}$ and PS Papaioannou ${ }^{1}$
}

1. Faculty of Veterinary Science, University of Thessaly, Karditsa, Greece 2. European Food Safety Authority (Task Force on Zoonoses) / Directorate General of Veterinary Services (Animal Health Directorate - Zoonoses Department), Ministry of Rural Development and Food, Athens,

Greece 3. Laboratory of Hygiene and Epidemiology, Faculty of Medicine, University of Thessaly, Larissa, Greece 4. Directorate General of Veterinary Services, Ministry of Rural Development and Food, Athens, Greece* Corresponding author email : vpapatsiros@yahoo.com Published online at www.veterinaryworld.org on 25-03-2011

\begin{abstract}
Streptococcus suis is a major porcine pathogen, causing economical health worldwide problems in the global swine industry. It is also emerging as a zoonotic agent capable of causing severe invasive disease in humans exposed to pigs or pork products. The most important clinical sign in swine and human is meningitis, but other pathological conditions have also been described. Serotype 2 is the most commonly associated with diseases in pigs and humans, and also the most frequently reported serotype isolated from diseased animals worldwide. The majority of human infection occurs in pork handlers, particularly in slaughterhouse workers and by minor wounds or skin abrasions contaminated by raw pork or viscera of pigs. Veterinarians should also be aware that a low but real risk may be present when manipulating S. suis-diseased animals that are probably shedding high numbers of this pathogen. Up today, in Greece there is no published epidemiological data for S. suis serotypes in swine herds and the zoonotic risk of S. suis infection in human with daily contact with pigs and pork meat. However, in our experience clinical forms of S. suis infection are common in most greek swine farms. The aim of this review study is to perform recent information about $\mathrm{S}$. suis infection in swine and human, focus on zoonotic risk of this emerging pathogen and prevention strategies.
\end{abstract}

Keywords: streptococcus suis, swine, human, emerging pathogen, zoonotic risk, pork meat

\section{I ntroduction}

Streptococcus suis (S. suis) is a major porcine pathogen endemic in all countries with a developed swine industry, causing great economic loss every year. Although $S$. suis is considered primarily a swine pathogen, it has been increasingly isolated from a wide range of animal species eg, cattle, sheep, goats, horses, including human beings, and birds, which suggests new concepts about some epidemiological aspects of the infection.

S. suis is s an $\alpha$-hemolytic streptococci related to Lancefield group, a gram-positive, encapsulated bacterium causes many different swine diseases. 35 different serotypes of $S$. suis have been described, along with subtypes defined as different genotypes within these serotypes (King et al. 2001). S. suis comprises of pathogenic, weakly pathogenic, and nonpathogenic strains (Gottschalk et al. 1999, Gottschalk and Segura 2000, Smith et al. 2001, King et al. 2001).
Various bacterial components such as extracellular and cell membrane-associated proteins have been suggested as virulence-associated factors, but their precise roles in the pathogenesis or virulence of $S$. suis have not been established (Gottschalk et al 1999, Gottschalk and Segura 2000). Serotype 2 of S. suis (SS2) is the most commonly associated with diseases in pigs and humans, and also the most frequently reported serotype isolated from diseased animals worldwide (Wisselink et al. 2000, Hill et al. 2005, Silva et al., 2006, Higgins and Gottschalk 1999). SS2 is the most prevalent type in clinical material from pigs in Europe (Wisselink et al. 2000) and the infection causes severe disease outbreaks in swine herds.

The epidemiology of $S$. suis occurrence among pigs is complex; it usually occurs as normal flora, but may be infectious or even lethal, especially among weaning piglets. Most clinically healthy pigs are carriers of multiple serotypes of $S$. suis The natural habitat of $S$. suis is the upper respiratory tract (particularly the tonsils and nasal cavities) (Gottschalk 
and Segura 2000), and the genital and alimentary tracts of pigs (Higgins and Gottschalk 1999). S. suis is transferred from vaginal secretions to the oral cavity of the piglet during parturition (Amass et al. 1996) and colonizes the tonsil soon after birth (Amass et al. 1995). Transmission between herds occurs usually by the movement of healthy carrier pigs. Their introduction into a non infected herd usually results in the subsequent onset of disease in weaners and/or growing pigs. However, some infected herds that show no illness may develop clinical disease in the presence of other predisposing factors, such as Porcine Reproductive and Respiratory Syndrome Virus (PRRSV). S. suis can also be transmitted via fomites and flies. The importance of other animal species or birds as reservoirs or vectors of the infection is unknown.

Although, the most important clinical manifestation in swine is meningitis, other pathological conditions have also been described, such as meningitis, endocarditis, septicemia with sudden death, arthritis and pneumonia, abortions, and abscesses (Gogolewski et al. 1990, Higgins and Gottschalk 1999, King et al. 2001, Smith et al. 2001, Huang et al. 2005, Gottschalk et al. 2007). Clinical infections are seen mainly in weaning or growing pigs and less frequently in suckling piglets. The earliest sign is usually fever, which may occur initially without other obvious signs. It is accompanied by a detectable bacteremia or pronounced septicemia, fluctuating fever, inappetence, depression, and shifting lameness (Touil et al. 1988). Even if meningitis / meningoen-cephalitis are the most striking clinical sign (on which a presumptive diagnosis is usually based), early nervous signs (such as incoordination, adoption of unusual stances, instability, paddling, opisthotonos, convulsions and nystagmus) are noticed (Reams et al. 1994, Higgins et al. 1990, Gottschalk and Segura 2000). Endocarditis is also a frequent clinical sign, accompanied by sudden death, dyspnea, cyanosis, and wasting. Septicemia, arthritis, and bronchopneumonia/ Pneumonia are less common signs and rhinitis, abortions, and vaginitis may also occur (Touil et al. 1988, Sanford and Higgins 1992, Higgins and Gottschalk 1999, Huang et al. 2005).

\section{Human infection - Zoonotic Risk}

It is recognized as a zoonotic agent since 1968 when the first case of meningitis reported in Denmark (Perch 1968). Since then the bacterium has caused sporadic human illness in other European countries (Watkins et al 2001, Pedroli et al. 2003, Manzin et al. 2008), as well as in Asia, such as in Thailand (Pootong
1993, BOE 2005, Rusmeechan and Sribusara 2008), in Hong Kong (Hui et al. 2005), in China (Ye et al. 2006) and Vietnam (Mai et al. 2008). Although human infection with $S$. suis has been occasionally documented in North America (Gottschalk et al. 2004), the first human case was not reported in the United States until 2006 (Willenburg et al. 2006). In Greece, the first case of $S$. suis septicemia and meningitis reported in 2005 (Mazokopakis et al. 2005). It was a case of septicemia caused by S22 complicated by meningitis and deafness in an elderly woman with history of prior contact with unprocessed pork meat. The causative agent was isolated from both the blood cultures and the cerebrospinal fluid. The patient's condition improved after treatment with penicillin, but deafness became permanent.

Occasionally it affects humans, particularly those occupationally exposed to pigs or pig products, causing meningitis, septicemia, endocarditis, toxic shock syndrome, arthritis, acute deafness, or other illnesses which are commonly fulminant and often fatal (Arends et al 1988, Bungener et al 1989, Peetermans et al 1989, Trottier et al 1991, Staats et al 1997, Ruoff et al 2003, Lun 2007, Wertheim et al 2009). A carriage state has been documented in pigs, healthy carriers being a source of $S$. suis transmission in herds, mainly through the respiratory route (Gottschalk et al 2007). As discussed in recent reports, the possibility cannot be excluded that humans may also be healthy carriers (Gottschalk et al 2007, Smith et al 2008, Wertheim et al 2009,) and that $S$. suis may become an opportunistic pathogen under particular circumstances such as stress, immunodeficiency or cancer (Gottschalk et al 2007, Manzin et al 2008). Meningitis with possible residual deafness is the most frequent clinical presentation of the infection in humans; septicaemia, pneumonia, endocarditis, arthritis and toxic shock syndrome have also been described (Colaert et al. 1986, Shneerson et al 1980). Outbreaks of SS 2 in humans were reported in People's Republic of China in 1998 and 2005 (WHO 2005). In these outbreaks 215 humans were infected and 38 of them died, mainly by streptococcal toxic shock syndrome (STSS) and some of them by meningitis or severe septicemia (Hu et al. 2000, Yu et al. 2006). This outbreak followed a pig die-off and the major risk factors were slaughtering a sick pig or handling the carcass of a pig that had died from unknown causes. In a recent study, the characteristics of 407 strains of $S$. suis isolated from diseased pigs investigated between 2003 - 2007 in China (Wei et al. 2009). Among the strains SS2 $(43.2 \%)$ was most prevalent with either 
systemic infection or pneumonia among all the serotypes, followed by serotypes $3(14.7 \%)$ and $4,8,5$, $7,1 / 2(3.2-6.4 \%)$. SS2 was more frequently isolated from swine with systemic infection, while serotype 3 was significantly associated with pneumonia.

People in daily contact with pigs (such as pig farmers, abattoir workers, persons transporting pork, meat inspectors, and butchers) are usually affected through the skin via a cut, infected wound or abrasion (Robertson and Blackmore 1989, Tarradas et al. 2001). The majority of human infection occurs in pork handlers, particularly in slaughterhouse workers and by minor wounds or skin abrasions contaminated by raw pork or viscera of pigs (Clifton-Hadley et al. 1984; Breton et al. 1986).

Recently, Zhu et al (2008) reported a case with a patient, who was exposed to pathogen in the contaminated water in the slaughterhouse, because he had not protected himself by wearing boots. Colonization of the nasopharynx, as observed in swine, or the gastrointestinal tract, suggested by observation of diarrhea as a prodromal sign, has also been suggested. Information about the occurrence and frequency of human colonization by $S$. suis is scarce, with most data coming from abattoir workers (Strangmann et al. 2002, Sala and Colombo 1989). The incubation period ranges from a few hours to 2 days (Lütticken et al. 1986). Manifestation of disease in pigs is not a prerequisite for infections in people in contact with pigs, since most animals are colonized by S. suis without clinical signs (Higgins and Gottschalk 1999). In addition to domestic pigs, wild boars may also be a source of $S$. suis infection for hunters and poachers (Halaby et al. 2000). From studies of contamination of hands and knives, it was concluded that eviscerators involved in removing the larynx and lungs from the carcasses have a significantly higher risk of exposure to $S$. suis than other abattoir workers (Breton et al 1986). Finally, one documented case in a veterinary surgeon has been reported (Walsh et al. 1992). In very few cases, there was no apparent connection with exposure to pigs or pork products (Strangmann et al. 2002). In addition, splenectomy, and to a lesser extent, alcoholism, have been suggested as important predisposing factors for development of serious S. suis disease (Watkins et al. 2001, Gallagher 2001).

In general, $S$. suis isolates from humans are phenotypically and genotypically similar to those recovered from pigs. In 2001, two cases of meningitis in a butcher and an abattoir worker who handled pork meat that originated from the same three closed farms were reported (Tarradas et al. 2001). Analysis of SS2 strains recovered from tonsils of healthy pigs from those farms were genotypically similar (but not identical) to the human strains. The slight differences between isolates were probably the consequence of adaptation to the new host or simply lack of reproducibility of the detection technique. Virulence properties of strains isolated from pigs or humans seem to be similar (Gottschalk and Segura 2000).

\section{Prevention}

Control and Prevention in swine: Affected pigs should be treated with injected antimicrobial for 3-5 days. Penicillin is the drug of choice although amoxicillin, ampicillin, cephalosporins, trimethoprim sulphonamide and other antimicrobials can be used but not the aminoglycosides or tetracyclines (Higgins and Gottschalk 1999). The sensitivity rate to amoxicillin and ampicillin is $\sim 90 \%$ and the use of ceftiofur hydrochloride can reduce mortality associated with PRRSV / S. suis coinfection (Schmitt et al. 2001). A high degree of resistance among $S$ suis isolates to some antibacterial agents such as tetracycline, clindamycin, erythromycin, kanamycin, neomycin, and streptomycin has been reported (Aarestrup et al. 1990, Escudero et al. 2007, Hendriksen et al. 2008). Adjunctive therapy with an anti-inflammatory agent is also recommended for treatment of $S$. suis meningitis in pigs. Animals with advance septicemia, meningitis and arthritis rarely respond and all cases should be re-assessed 3 days after the onset of treatment. Pigs in the same pen as affected animals should be treated in feed or in water. Control of the disease in older pigs can be by medication of the water with ampicillin or amoxicillin with phenoxymethyl penicillin or procaine penicillin at $75-100 \mathrm{~g} / \mathrm{tn}$ at $200-300 \mathrm{~g} / \mathrm{tn}$ in feed for 7 days prior to expected disease. Penicillins should be orally administered through drinking water to reduce the interference in absorption due to feed. Amoxicillin has advantages over natural penicillins for mass medication-its body clearance is lower than that of penicillin $\mathrm{V}$, and higher serum concentrations are obtained (Higgins and Gottschalk 1999).

Control of the disease in piglets may be possible by farrowing sows in isolation and by fumigating the accommodation between animals. Injection of piglets at birth with long-acting penicillin may prevent disease. All-in, all-out husbandry methods can reduce the spread of infection. Medicated early weaning has eradicated disease from a herd but slaughter, disinfection and repopulation is most reliable (Higgins 
and Gottschalk 1999). Up today commercial or autogenous bacterins vaccines have been used, but their efficacy is under discussion (Wisselink et al 2000, Baums et al 2009).

Prevention of Human infection: While workers handling fresh pork products in slaughterhouse need awareness, more evidences from recent reports show that pig breeder, butchers and any persons who handle or eat uncooked pork products can be at high risk of infection by S. suis (Martin et al 2001, (Donsakul et al 2003). The $S$. suis meningitis usually responds very well to treatment with large doses of intravenous penicillin. Rarely the organism is resistant to penicillin. Nevertheless within 24 hours of treatment, the fever, and the headache and the neck stiffness often resolve, and deafness is usually permanent.

Several investigators have suggested that the infrequent diagnosis of human $S$. suis infection is due to under diagnosis or misdiagnosis, rather than a true absence of disease (Gottschalk et al 2004, Donsakul et al 2003, Heidt et al 2005). Diagnosis of S. suis infection is based on characteristic macroscopical lesions, bacterial cultures, and biochemical and serological characterization (Durand et al 2001). Early diagnosis and effective antibiotic therapy are key points for increasing the survival rate. S. suis strains isolated from diseased pigs are most susceptible to $\beta$ lactam antimicrobials, aminoglycosides, enrofloxacin, novobiocin and spectinomycin and more than $87 \%$ of the $S$. suis isolates are resistant to tetracyclines, sulphonamides, macrolides and clindamycin (Vela et al 2005). Recently, in Italy high genetic diversity among $59 \mathrm{~S}$. suis strains ( 2 recent human strains and 57 swine clinical isolates from different herds and regions) were observed and the majority of which were resistant to macrolides $(78 \%)$ and tetracyclines (90\%) (Princivalli et al. 2009). In another study, a high level of resistance to tetracycline $(48.0-92.0 \%)$, trimethoprim-sulphonamide (3.0 to $51.5 \%)$ and erythromycin $(29.1-75.0 \%)$ was observed in many European countries (Rene et al 2008).

As there is no $S$. suis vaccine for humans, suitable preventive measures and supervision of high risk population are critically important to decrease the transmission of $S$. suis to humans.

All medical personnel should be aware of $S$.suis infection, especially when streptococcal meningitis is diagnosed in persons working with pigs or pork products. Veterinarians should also be aware that a low but real risk may be present when manipulating $S$. suis-diseased animals that are probably shedding high numbers of this zoonotic etiological agent. It is suggested that the appropriate handling of fresh pork products can prevent the infection and early diagnosis with early treatment may prevent disabling deafness. Generally, people with close contact to pigs or unprocessed pork have to observe rules of personal and environmental hygiene, which include:

- avoiding generating skin lesions during meat processing procedures should be taken covering open wounds with water-proof dressing

washing hands, arms and other exposed parts of the body through after each contact with pigs or pork meat

- $\quad$ keeping the uncooked pork apart from other cooked food

- $\quad$ cooking pork to an temperature of $70{ }^{\circ} \mathrm{C}$ or until the juice is clear and not pink

- consulting a doctor in case of febrile illness after exposure to pigs or pork meat.

\section{References}

1. Aarestrup FM, Rasmussen SR, Artursson K, Jensen NE (1998): Trends in the resistance to antimicrobial agents of Streptococcus suis isolates from Denmark and Sweden. Vet Microbiol., 63(1): 71-80.

2. Amass SF, Clark LK, Knox K, Wu CC, Hill MA (1996): Streptococcus suis colonization of piglets during parturition. Swine Health Prod.; 4: 269-272.

3. Amass SF, Clark LK, Wu CC. (1995): Source and timing of Streptococcus suis infection in neonatal pigs: implications for early weaning procedures. Swine Health Prod.; 3: 189-193.

4. Baums CG, Kock C, Beineke A, Bennecke K, Goethe R, Schroder C et al. (2009): Streptococcus suis Bacterin and Subunit Vaccine Immunogenicities and Protective Efficacies against Serotypes 2 and 9. Clin Vaccine Immunol: 200-208.

5. Breton J, Mitchell WR, Rosendal S. (1986): Streptococcus suis in slaughter pigs and abattoir workers. Can J Vet Res, 50: 338-341.

6. Clifton-Hadley FA, Alexander TJ, Enright MR, Guise J. (1984) Monitoring herds for Streptococcus suis type 2 by sampling tonsils of slaughter pigs. Vet Rec., 115: 562-4.

7. Colaert J, Allewaert M, Magerman H, Vandeven J, Vandepitte J. (1986): Streptococcus suis meningitis in man. Deafness after meningitis. Lancet, 1: 134-135.

8. Donsakul K, Charungthai D, Witoonpanich R. (2003): Streptococcus suis: clinical features and diagnostic pitfalls. Southeast Asian J Trop Med Public Health, 34: 154-158.

9. Durand F, Perino CL, Recule C, Brion JP, Kobish M, Guerber F, et al. (2001): Bacteriological diagnosis of Streptococcus suis meningitis. Eur J Clin Microbiol Infect Dis, 20: 519-21.

10. Escudero JA, Millan AS, Catalan A, de la Campa AG, Rivero E, Lopez G et al. (2007): First Characterization of Fluoroquinolone Resistance in Streptococcus suis. Antimicrobial Agents and Chemotherapy, 51: 777-782.

11. Gallagher F. Streptococcus suis infection and splenectomy. Lancet. 2001; 357: 1131-1132.

12. Gogolewski RP, Cook RW, O'Connell CJ. (1990): 
Streptococcus suis serotypes associated with disease in weaned pigs. Aust Vet J, 67: 202-204.

13. Gottschalk M, Higgins R, Quessy S. (1999): Dilemma of the virulence of Streptococcus suis strains. J Clin Microbiol, 37 : 4202-4203.

14. Gottschalk M, Segura M, Xu J. (2007): Streptococcus suis infections in humans: the Chinese experience and the situation in North America. Anim Health Res Rev, 8(1): 2945.

15. Gottschalk M, Segura M. (2000): The pathogenesis of the meningitis caused by Streptococcus suis: Unresolved questions. Vet Microbiol, 76: 259-272.

16. Gottschalk M. (2004): Porcine Streptococcus suis strains as potential sources of infection in humans: an underdiagnosed Problem in North America? Journal of Swine Health Prod 12:197-199.

17. Halaby T, Hoitsma E, Hupperts R, Spanjaard L, Lurink M, Jacobs J. (2000): Streptococcus suis meningitis, a poacher's risk. Eur J Clin Microbiol Infect Dis, 19: 943-945.

18. Heidt MC, Mohamed W, Hain T, Vogt PR, Chakraborty T, Domann E. (2005): Human infective endocarditis caused by Streptococcus suis serotype 2. J Clin Microbiol, 43: 4898-4901.

19. Hendriksen RS, Mevius DJ, Schroeter A, Teale C, Jouy E, Butaye P et al. (2008): Occurrence of antimicrobial resistance among bacterial pathogens and indicator bacteria in pigs in different European countries from year 2002 2004: the ARBAO-II study. Acta Vet Scand, 13(1): 50: 19

20. Higgins R, Gottschalk M, Mittal KR, Beaudoin M. (1990): Streptococcus suis Infection in Swine.A Sixteen Month Study. Can J Vet Res, 54: 170-173.

21. Higgins R, Gottschalk M. (2000): Distribution of Streptococcus capsular types in 1999. Can Vet J, 41: 414.

22. Higgins R, Gottschalk M. (1999): Streptococcal diseases. In: Straw BE, D'Allaire S, Mengeling WL, Taylor DJ, eds. Diseases of Swine. Ames, Iowa: Iowa State University Press;:563-578.

23. Hill JE, Gottschalk M, Brousseau R, Harel J, Hemmingsen SM, Goh SH. (2005): Biochemical analysis, cpn60 and 16S rDNA sequence data indicate that Streptococcus suis serotypes 32 and 34, isolated from pigs, are Streptococcus orisratti. Vet Microbiol, 107: 63-69.

24. Hu X, Zhu F, Wang H, Chen S, Wang G, Sun J, et al. (2000): Studies on human streptococcal infectious syndrome caused by infected pigs. Zhonghua, 34: 150-152.

25. Huang YT, Teng LJ, Ho SW, Hsueh PR. (2005): Streptococcus suis infection. J Microbiol Immunol Infect, 38: 306-313.

26. Hui AC, Ng KC, Tong PY, Mok V, Chow KM, Wu A, et al. (2005): Bacterial meningitis in Hong Kong: 10-years' experience. Clin Neurol Neurosurg, 107: 366-370.

27. King SJ, Heath PJ, Luque I, Tarradas C, Dowson CG, et al. (2001): Distribution and genetic diversity of suilysin in Streptococcus suis isolated from different disease of pigs and characterization of the genetic basis of suilysin absence. Infect Immun, 69: 7572-7582.

28. Lun ZR, Wang QP, Chen XG, Li AX, Zhu XQ. Streptococcus suis: an emerging zoonotic pathogen. Lancet Infect Dis. 2007; 7(3): 201-209.

29. Lütticken R, Temme N, Hahn E, Bartelheimer EW. (1986): Meningitis caused by Streptococcus suis: case report and review of the literature. Infection, 14: 181-185.

30. Mai NT, Hoa NT, Nga TV, Linh LD, Chau TT, Sinh DX, et al. (2008): Streptococcus suis meningitis in adults in Vietnam. Clin Infect Dis, 46: 659-667.
31. Manzin A, Palmieri C, Serra C, Saddi B, Princivalli MS, Loi $\mathrm{G}$ et al. Streptococcus suis meningitis without evidence of animal contact, Italy. Emerg Infect Dis. 2008; 14(12): 19461948

32. Martin $\mathrm{CH}$, Walid M, Torsten H, Vogt PR, Trinad C and Eugen D. (2005): Human Infective Endocarditis Caused by Streptococcus suis Serotype 2. J Clin Microbiol, 43(90): 4898-4901.

33. Mazokopakis EE, Kofteridis DP, Papadakis JA, Gikas AH and Samonis GJ. (2005): First case report of Streptococcus suis septicaemia and meningitis from Greece. European Journal of Neurology, 12(6): 487-489.

34. Pedroli S, Kobisch M, Beauchet O, Chaussinand JP, Lucht F. (2003): Bactériémie à Streptococcus suis [Streptococcus suis bacteremia]. La Presse medical, 32: 599-601.

35. Peetermans WE, Moffie BG, Thompson J. (1989): Bacterial endocarditis caused by Streptococcus suis type 2. J Infect Dis, 159: 595-596.

36. Perch B, Kristjansen P, Skadhauge KN. Group R streptococci pathogenic for man. (1968):Two cases of meningitis and one fatal case of sepsis. Acta Pathol Microbiol Scand B, 74: 69-76.

37. Pootong P, Boongrid P, Phuaprsdit P. Phuaprsdit P. (1993): Streptococcus suis meningitis at Ramathibodi Hospital. Ramathibodi Med J, 16:203-207.

38. Princivalli MS, Palmieri C, Magi G, Vignaroli C, Manzin A Camporese A et al. (2009): Genetic diversity of Streptococcus suis clinical isolates from pigs and human in Italy (2003-2007). Euro Surveill, 14 (33).

39. Reams RY, Lawrence T. Glickman, Harrington DD, Thacke HL, Bowersock TL. (1994): Streptococclcs suis infection in swine: a retrospective study of 256 cases. Part II. Clinical signs, gross and microscopic lesions, and coexisting microorganisms. J Vet Diagn Invest, 6: 326-334.

40. Rene HS, Mevius DJ, Schroeter A, Teale C, Jouy E, Butaye P et al. (2008): Occurrence of antimicrobial resistance among bacterial pathogens and indicator bacteria in pigs in differen European countries from year 2002 - 2004: the ARBAO-II study. Acta Vet Scan, 50: 19.

41. Robertson ID, Blackmore DK. Occupational exposure to Streptococcus suis type 2. 1989; Epidemiol Infect, 103: 157-164.

42. Robertson ID, and DK Blackmore. (1989): Occupational exposure to Streptococcus suis type 2. Rev Infect Dis, 101:1102-1105

43. Ruoff KL, Whiley RA, Beighton D. (2003): Streptococcus In P. R. Murray, E. J. Baron, M. A. Pfaller, J. H. Jorgenson, and R. H. Yolken (ed.), Manual of clinical microbiology, 8th ed. ASM Press, Washington, D.C.: 405-421.

44. Rusmeechan S, Sribusara P. (2008): Streptococcus suis meningitis: the newest serious infectious disease. $\mathrm{J} \mathrm{Med}$ Assoc Thai, 91(5): 654-658.

45. Sala V, Colombo A. (1989): Infection risks of Streptococcus suis type 2 localizations in slaughtered swine. Arch Vet It, 40:180-184.

46. Schmitt CS; Halbur PG, Roth JA, Kinyon JM, Kasorndorkbua C, Thacker B. Influence of ampicillin, ceftiofur, attenuated live PRRSV vaccine, and reduced dose Streptococcus suis exposure on disease associated with PRRSV and S. suis coinfection. Vet microbial. 2001; 78(1): 29-37.

47. Shneerson JM, Chattopadhyay B, Murphy MF, Fawcett IW (1980): Permanent perceptive deafness due to Streptococcus suis type II infection. J Laryngol Otol, 94: 425-427.

48. Silva L, Baums CG, Rehm T, Wisselink H, Goethe R, 
Valentin-Weigand P. (2006): Virulence-associated gene profiling of Streptococcus suis isolates by PCR. Vet Microbiol, 155: 117-127.

49. Smith HE, Buijs H, Wisselink HJ, Stockhofe-Zurwieden N, Smits MA. (2001): Selection of virulence-associated determinants of Streptococcus suis serotype 2 by in vivo complementation. Infect Immun, 69: 1916-1966.

50. Smith TC, Capuano AW, Boese B, Myers KP, Gray GC. (2008): Exposure to Streptococcus suis among US swine workers. Emerg Infect Dis, 14(12): 1925-1927.

51. Strangmann E, Fröleke H, Kohse KP. (2002): Septic shock caused by Streptococcus suis: case report and investigation of a risk group. Int J Hyg Environ Health, 205: 385-392.

52. Tarradas C, Luque I, de Andres D, Abdel-Aziz Shahein YE, Pons P, Gonzalez F, et al. (2001): Epidemiological relationship of human and swine Streptococcus suis isolates. $J$ Vet Med B, 48: 347-355.

53. Touil F, Higgins R, Nadeu M. (1988): Isolation of Streptococcus suis from diseased pigs in Canada. Vet Microbiol, 17:171-177.

54. Trottier S, Higgins R, Brochu G, Gottschalk M. (1991): A case of human endocarditis due to Streptococcus suis in North America. Rev Infect Dis, 13: 1251-1252.

55. Vela AI, Moreno MA, Cebolla JA, González S, Latre MV, Land D et al. (2005): Antimicrobial susceptibility of clinical strains of Streptococcus suis isolated from pigs in Spain . Vet Microbiol, 105 (2): 143-147.

56. Walsh B, Williams AE, Satsangi J. (1992): Streptococcussuis type 2: pathogenesis and clinical disease. Rev MedMicrobiol, 3: 65-71.
57. Watkins EJ, Brooksby P, Schweiger MS, Enright SM. (2001): Septicaemia in a pig-farm worker. Lancet, 357: 38.

58. Wei Zigong Ran Li, Anding Zhang, Hongkui He, Yafeng Hua, Jing Xia, Xuehui Cai, et al. (2009): Characterization of Streptococcus suis isolates from the diseased pigs in China between 2003 and 2007. Vet Microbiol, 137: 196-201.

59. Wertheim HF, Nghia HD, Taylor W, Schultsz C. (2009): Streptococcus suis: an emerging human pathogen. Clin Infect Dis, 48(5):617-625.

60. Willenburg KS, Sentochnik DE, Zadoks RN. (2006): Human Streptococcus suis meningitis in the United States. $N$ Engl J Med, 354: 1325.

61. Wisselink HJ, Vecht U, Smith HE, Stockhofe-Zurwieden N, Peperkamp K, Vecht U. (2000): Distribution of capsular types and production of muramidase-released protein (MRP) and extracellular factor (EF) of Streptococcus suis strains isolated from diseased pigs in seven European countries. Vet. Microbiol, 74: 237-248.

62. World Health Organization. (2005): Outbreak associated with Streptococcus suis in pigs, China. Wkly Epidemiol Rec, 80: 269-270.

63. Ye C, Zhu X, Jing H, Du H, Segura M, Zheng H et al. (2006): Streptococcus suis sequence type 7 outbreak, Sichuan, China. Emerg Infect Dis, 12(8): 1203-1208.

64. Yu H, et al. (2006): Human Streptococcus suis outbreak, Sichuan, China. Emerg Infect Dis, 12: 914-920.

65. Zhu Y, Tana Z, Zhub L, Pana H, Zhangb X, Xub L et al. (2008): A Streptococcus suis serotype 2 caused streptococcal toxic shock syndrome(STSS) in a patient. JNMU, 22(5): 313-316. 